\title{
Development and Evaluation of GlycanDock: A Protein-Glycoligand Docking Refinement Algorithm in Rosetta
}

Morgan L. Nance ${ }^{1}$, Jason W. Labonte ${ }^{2,3,4}$, Jared Adolf-Bryfogle ${ }^{5,6,7}$, Jeffrey J. Gray ${ }^{1,2, *}$

1 Program in Molecular Biophysics, Johns Hopkins University, Baltimore, Maryland 21218, United States

2 Department of Chemical and Biomolecular Engineering, Johns Hopkins University, Baltimore, Maryland 21218, United States

${ }^{3}$ Department of Chemistry, Franklin \& Marshall College, Lancaster, Pennsylvania 17603, United States

${ }^{4}$ Department of Chemistry, Gettysburg College, Gettysburg, Pennsylvania 17325, United States

5 Protein Design Lab, Institute for Protein Innovation, Boston, Massachusetts 02115, United States

${ }^{6}$ Division of Hematology/Oncology, Boston Children's Hospital, Boston, Massachusetts 02115, United States

${ }^{7}$ Department of Pediatrics, Harvard Medical School, Boston, Massachusetts 02115, United States

${ }^{*}$ Correspondence to:

Jeffrey J. Gray

Johns Hopkins University

$3400 \mathrm{~N}$ Charles St

Chemical and Biomolecular Engineering

Baltimore, Maryland 21218

Office: Maryland Hall 208

Phone: 410-516-5313

Email: jgray@jhu.edu 
1GWM 2 Å ring-RMSD

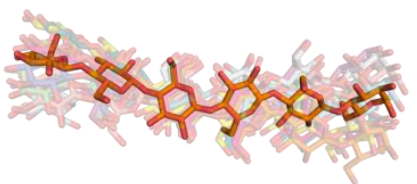

1GWM 4 Å ring-RMSD

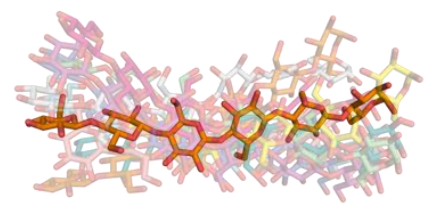

1GWM 6 Å ring-RMSD

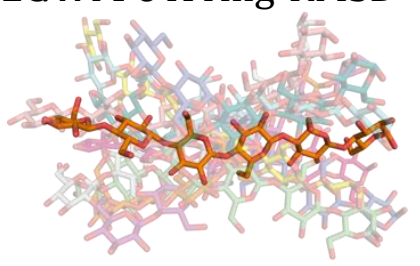

1GWM 8 Å ring-RMSD

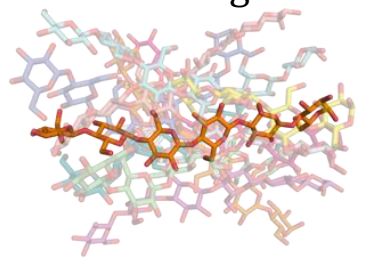

1GWM 10 Å ring-RMSD

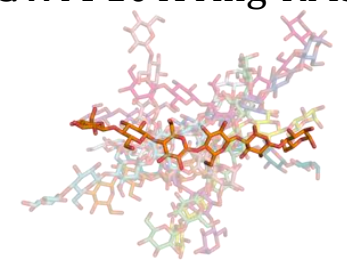

2OVU 2 Å ring-RMSD

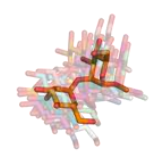

2OVU 4 Å ring-RMSD

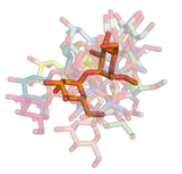

2OVU 6 Å ring-RMSD

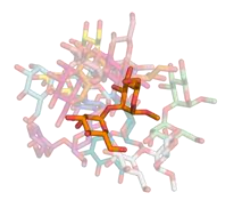

2OVU 8 Å ring-RMSD

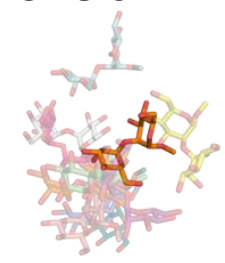

20VU 10 Å ring-RMSD

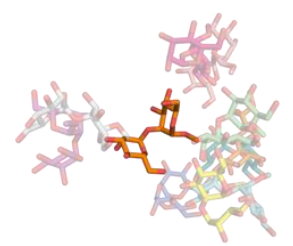

1MFE $2 \AA$ Ång-RMSD

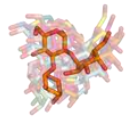

1MFE 4 Å ring-RMSD

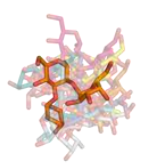

1MFE 6 Å ring-RMSD

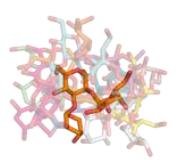

1MFE 8 Å ring-RMSD

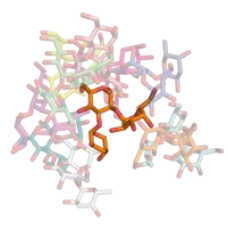

1MFE 10 Å ring-RMSD

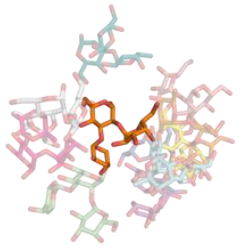

Figure S1: Example conformations of a glycoligand in its native bound state (orange, solid sticks) compared to randomly perturbed conformations of increasing ring-RMSD (colored, transparent sticks) for three benchmark targets (a hexasaccharide, a disaccharide, and a trisaccharide). Protein backbone omitted for clarity. 


\section{Bound Benchmark Target Set}
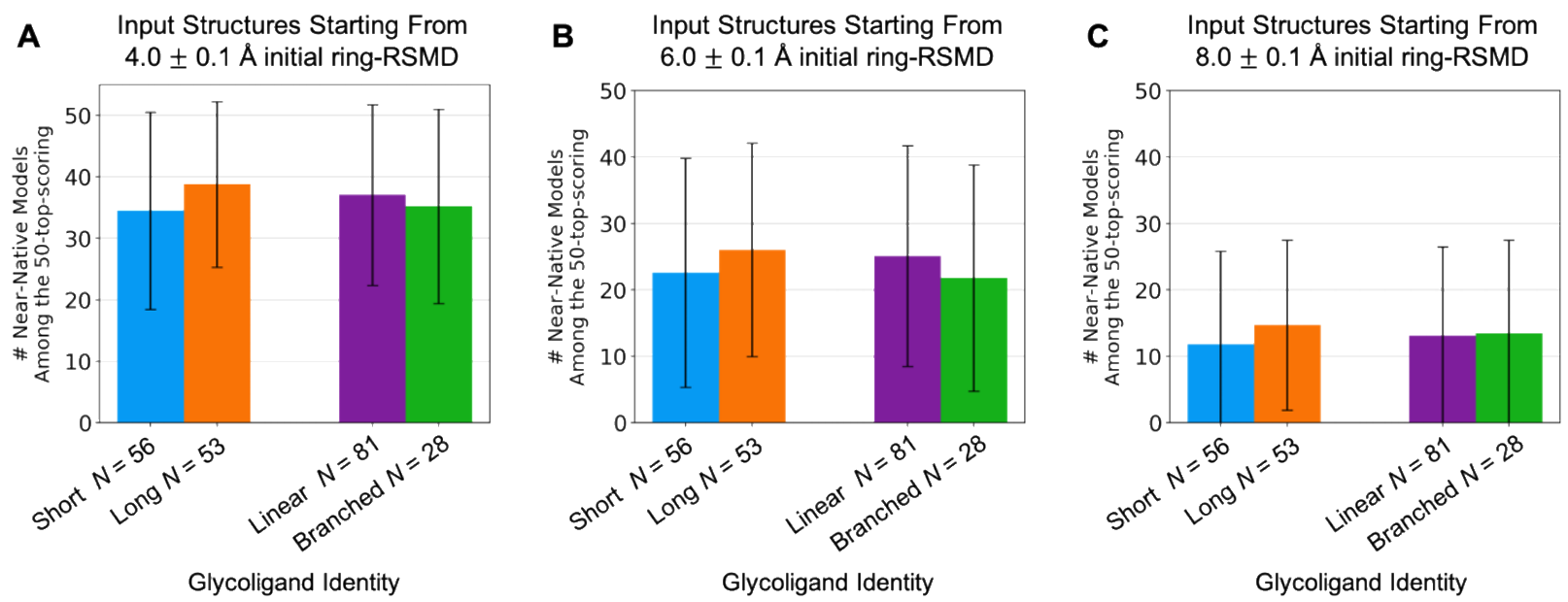

Unbound Benchmark Target Set

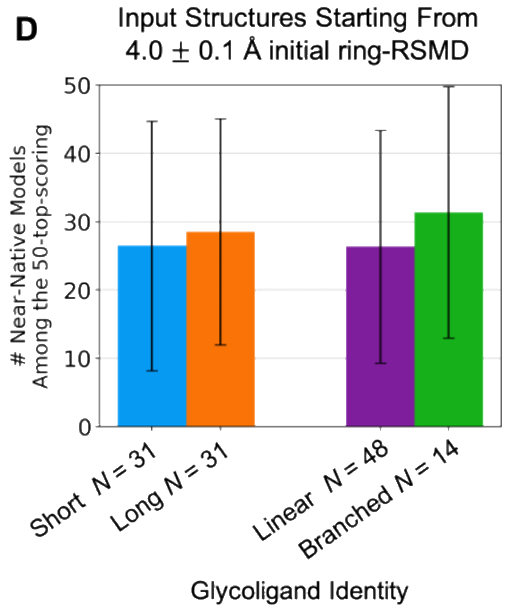

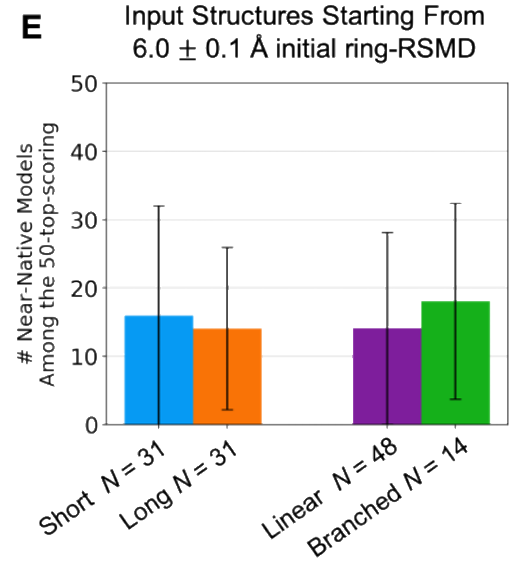

Glycoligand Identity

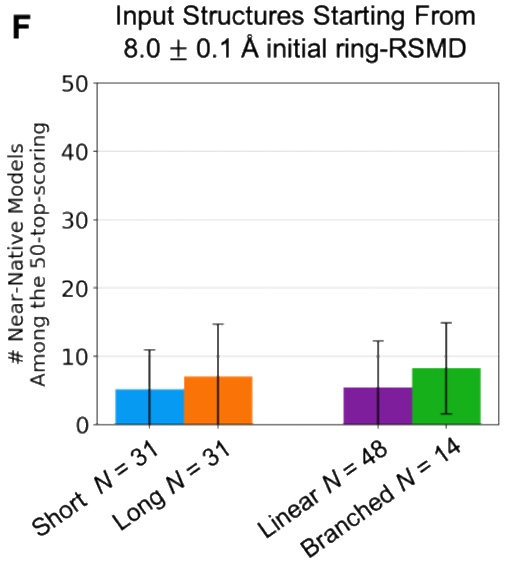

Glycoligand Identity

Figure S2: Comparison of the average count of near-native models among the 50-topscoring models from the bound $(N=109)$ and unbound $(N=62)$ benchmark target set as grouped by the glycoligand identity. (A) The average count of near-native models among the 50-top-scoring models of each target from the bound benchmark set as grouped either by the size of the glycoligand or whether the glycoligand is linear or has branched connections. The average count of near-native models among the 50-top-scoring models of the 56 targets with short glycoligands (blue bar; di- and trisaccharides) is compared to the 53 targets with long glycoligands (orange bar, tetrasaccharides and longer). Similarly, the average count of near-native models among the 50-top-scoring models of the 81 targets with linear glycoligands (purple bar) is compared to the 28 targets with glycoligands with branched connections (green bar). The error bar represents the standard deviation. All input starting structures were $4.0 \pm 0.1 \AA$ initial ring-RMSD. (B) and (C) Same as (A), but for input starting structures of $6.0 \pm 0.1 \AA$ and $8.0 \pm 0.1$ initial ring-RMSD, respectively. (D), (E), and (F) Same as (A), (B), and (C) but from targets from the unbound benchmark set. 

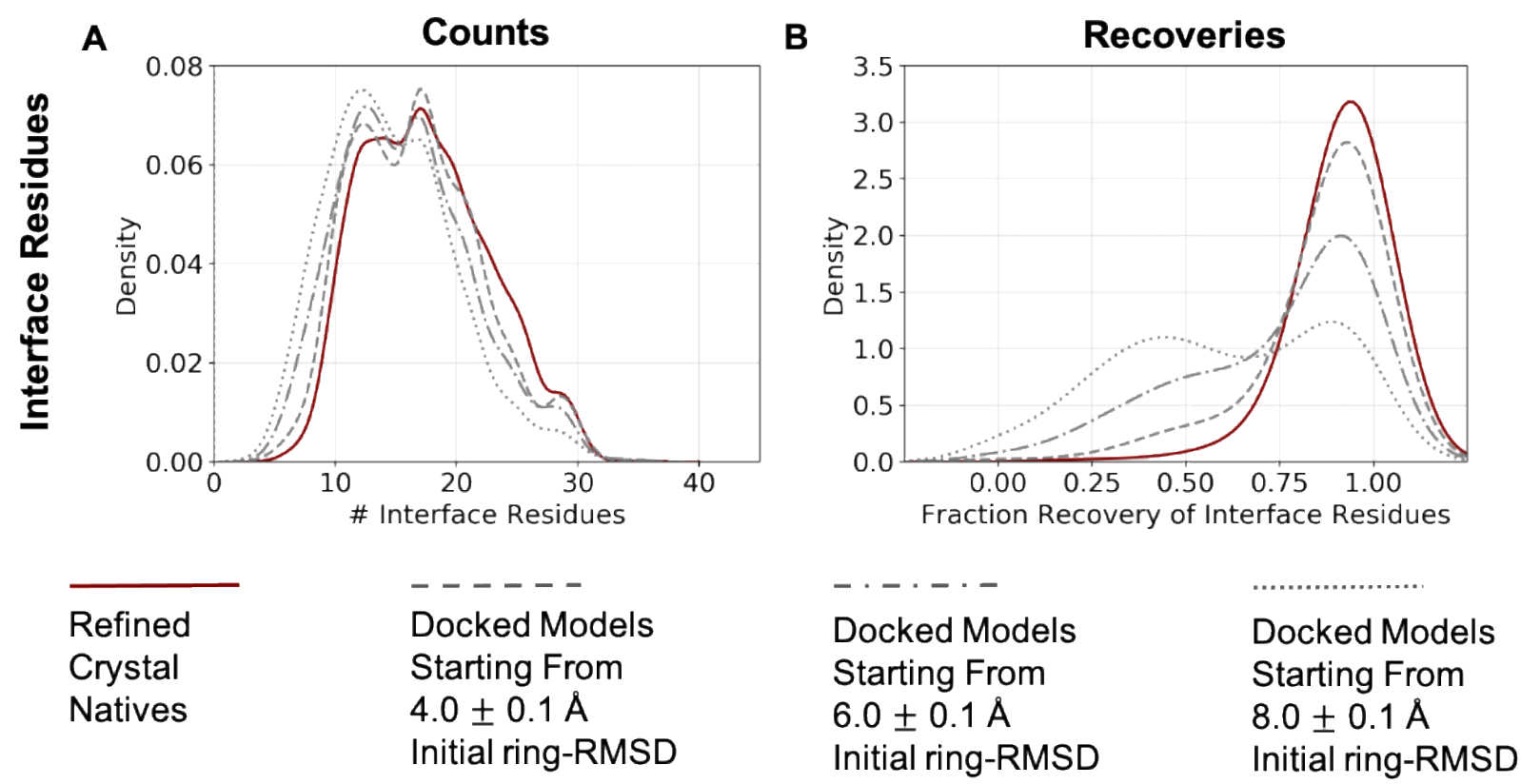

Figure S3: Counts and recovery of interface residues by the 50-top-scoring GlycanDock models. (A) Distributions of the count of interface residues by the 50-top-scoring GlycanDock models per target predicted from input structures of 4.0, 6.0, and 8.0 A initial ring-RMSD (gray dashed, dash-dot, and dotted lines, respectively). The distribution of the count of interface residues after GlycanDock crystal refinement (solid, maroon) serves as a reference. (B) Distributions of the recovery of native interface residues by the 50-top-scoring GlycanDock models per target. Discrete data are smoothed using kernel density fits using Seaborn ${ }^{1}$ (kdeplot), resulting in some curves extending below fractions of 0.0 and above 1.0. A bin width of 0.5 was used to fit the counts of native interface residues, and a bin width of 0.1 was used to fit the recoveries. 


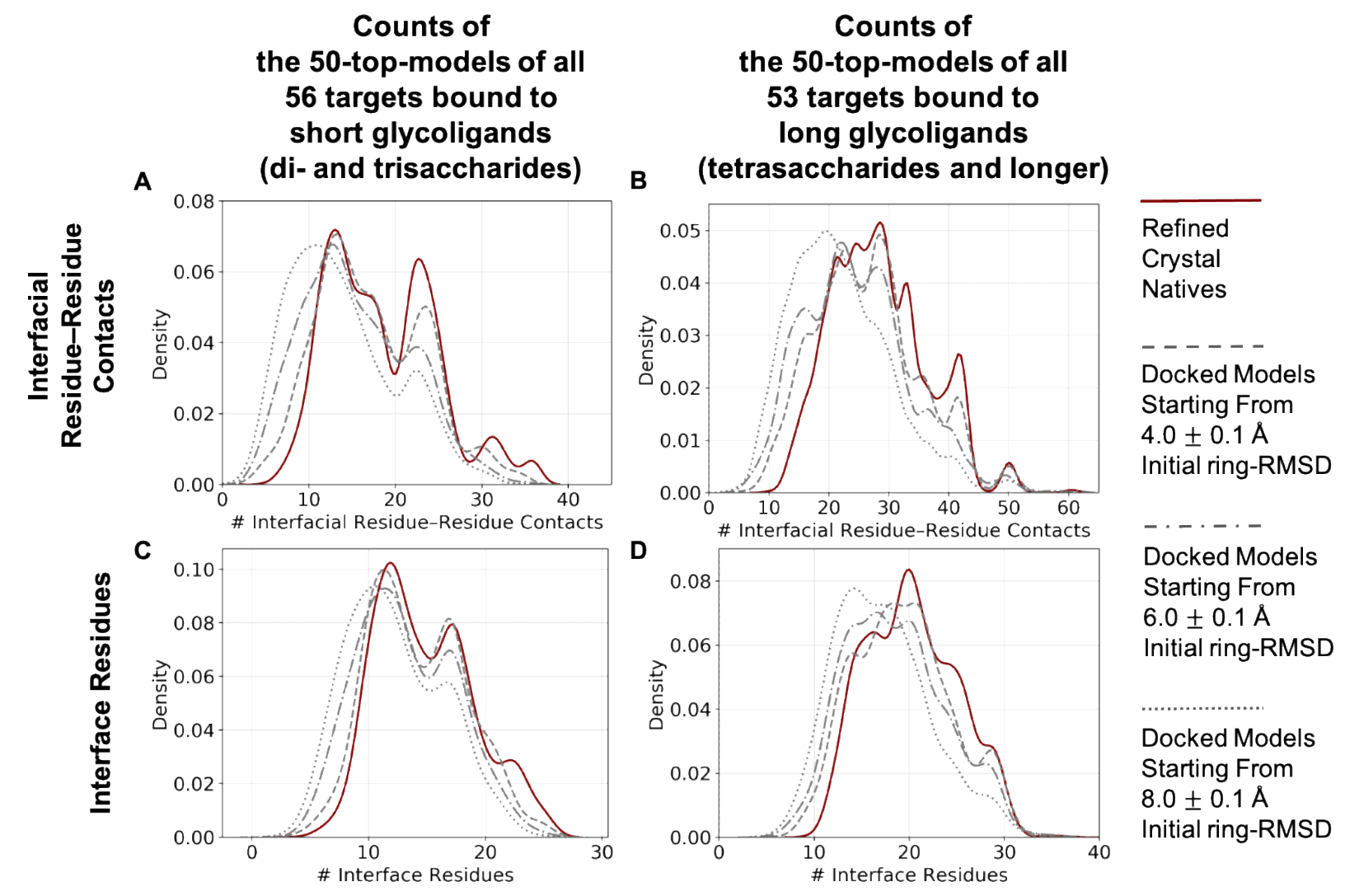

Figure S4: Counts and recovery of interfacial residue-residue contacts and interface residues by the 50-top-scoring GlycanDock models per target grouped based on glycoligand length. (See Figure 4 and S3 for more details). 
Table S1: GlycanDock and relevant Rosetta flags, descriptions, and default values. †Flags are GlycanDock specific and should be prefixed with "-carbohydrates:glycan_dock:" (e.g., -carbohydrates:glycan_dock:n_repeats 3). All accepted Rosetta flags can be found at https://www.rosettacommons.org/docs/latest/full-options-list.

\begin{tabular}{|c|c|c|}
\hline Flag & Description & Default \\
\hline refine_only ${ }^{\dagger}$ & $\begin{array}{l}\text { Skip the Stage } 1 \text { initial perturbation and employ only the uniform } \pm 15^{\circ} \text { glycosidic } \\
\text { torsion angle perturbations using the SmallBBSampler during Stage } 2 \text { docking } \\
\text { and refinement }\end{array}$ & False \\
\hline prepack_only ${ }^{\dagger}$ & Perform only Stage 0 pre-packing & False \\
\hline stage1_rotate_glycan_about_com ${ }^{\dagger}$ & $\begin{array}{l}\text { During Stage } 1 \text { initial perturbation, randomly rotate the glycoligand about its } \\
\text { center-of-mass in } 360^{\circ} \text { space }\end{array}$ & False \\
\hline stage1_perturb_glycan_com_trans_mag ${ }^{\dagger}$ & $\begin{array}{l}\text { The translational magnitude of the Gaussian perturbation on the glycoligand's } \\
\text { center-of-mass performed during Stage } 1\end{array}$ & $0.5 \AA$ \\
\hline stage1_perturb_glycan_com_rot_mag ${ }^{\dagger}$ & $\begin{array}{l}\text { The rotational magnitude of the Gaussian perturbation on the glycoligand's } \\
\text { center-of-mass performed during Stage } 1\end{array}$ & $7.5^{\circ}$ \\
\hline stage1_torsion_uniform_pert_mag ${ }^{\dagger}$ & The magnitude of the uniform perturbation on each glycosidic torsion angle & $12.5^{\circ}$ \\
\hline n_repeats ${ }^{\dagger}$ & $\begin{array}{l}\text { Number of times to repeat Stage } 2 \text { of GlycanDock if the final model does not have } \\
\text { a negative interface score }\end{array}$ & 3 \\
\hline n_rigid_body_rounds ${ }^{\dagger}$ & The number of inner cycles of rigid-body perturbations to perform & 8 \\
\hline slide_glycan_into_contact ${ }^{\dagger}$ & $\begin{array}{l}\text { Allow the glycoligand's center-of-mass to be periodically translated toward the } \\
\text { protein receptor's center-of-mass during rigid-body sampling }\end{array}$ & True \\
\hline n_torsion_rounds ${ }^{\dagger}$ & The number of inner cycles of glycosidic torsion perturbations to perform & 8 \\
\hline stage2_trans_mag ${ }^{\dagger}$ & $\begin{array}{l}\text { During Stage } 2 \text { docking and refinement, this is the magnitude for performing a } \\
\text { translational Gaussian perturbation on the glycoligand's center-of-mass. }\end{array}$ & $0.5 \AA$ \\
\hline stage2_rot_mag ${ }^{\dagger}$ & $\begin{array}{l}\text { During Stage } 2 \text { docking and refinement, this is the magnitude for performing a } \\
\text { rotational Gaussian perturbation on the glycoligand's center-of-mass. }\end{array}$ & $7.5^{\circ}$ \\
\hline full_packing_frequency ${ }^{\dagger}$ & $\begin{array}{l}\text { When packing after each sampling perturbation in Stage } 2 \text {, at what frequency } \\
\text { should the PackRotamersMover be called? Set to } 0 \text { to use only the } \\
\text { EnergyCutRotamerTrialsMover after every perturbation. Set to the value of } \\
\text { n_rigid_body_rounds and n_torsion_rounds to use the PackRotamersMover only } \\
\text { after the last perturbation }\end{array}$ & 8 \\
\hline $\mathrm{mc}_{-} \mathrm{kt}^{\dagger}$ & $\begin{array}{l}\text { During Stage } 2 \text { docking and refinement, the value of kT used to accept or reject } \\
\text { moves based on the Metropolis criterion. }\end{array}$ & 0.6 \\
\hline -include_sugars & $\begin{array}{l}\text { Include carbohydrate parameters from the database when initializing Rosetta. } \\
\text { This flag is required to use Rosetta with any inputs that include carbohydrates }\end{array}$ & $\begin{array}{l}\text { False (must be } \\
\text { set to True) }\end{array}$ \\
\hline
\end{tabular}




\begin{tabular}{|c|c|c|}
\hline -alternate_3_letter_codes & $\begin{array}{l}\text { When initializing Rosetta with carbohydrate database parameters, include } \\
\text { loading the three-letter PDB codes (e.g., GLC, MMA, SIA) for reading.pdb files }\end{array}$ & $\begin{array}{l}- \text { (must be set to } \\
\text { pdb_sugar for } \\
\text { PDB inputs) }\end{array}$ \\
\hline -auto_detect_glycan_connections & $\begin{array}{l}\text { Sets whether to search for non-protein connections using PDB coordinates and } \\
\text { generate LINK records }\end{array}$ & $\begin{array}{l}\text { False } \\
\text { (recommended } \\
\text { to set to True) } \\
\end{array}$ \\
\hline -lock_rings & $\begin{array}{l}\text { Allow alternative ring conformations to be sampled. Only low-energy conformers } \\
\text { will be sampled, if known. Otherwise, all ideal ring conformers will be sampled }\end{array}$ & $\begin{array}{l}\text { True } \\
\text { GlycanDock, } \\
\text { specifically) }\end{array}$ \\
\hline -docking:partners & Defines docking partners by chain ID. Example: docking chain $\mathrm{X}$ to $\mathrm{L}+\mathrm{H}$ is " $\mathrm{LH} \mathrm{X}^{\mathrm{X}}$ & $\begin{array}{l}\text { (must } \\
\text { provided) }\end{array}$ \\
\hline -n_cycles & The number of outer cycles controlling the Stage 2 score ramping procedure & $\begin{array}{l}10 \text { (in } \\
\text { GlycanDock, } \\
\text { specifically) }\end{array}$ \\
\hline -nstruct & The number of models to generate using the GlycanDock algorithm & 1 \\
\hline -auto_setup_metals & $\begin{array}{l}\text { Automatically adds covalent linkages to bound metal ions, as well as atom pair } \\
\text { constraints and angle constraints to hold ions in place, on PDB import. Also, sets } \\
\text { the atom_pair_constraint and angle_constraint weights in the default score } \\
\text { function to 1.0. }\end{array}$ & $\begin{array}{l}\text { False (set to } \\
\text { True if bound } \\
\text { ions are in the } \\
\text { system) }\end{array}$ \\
\hline
\end{tabular}


Table_S2.xls: Information on the 109 protein-glycoligand crystal structures employed as the bound benchmark target set including each structure's relevant chain IDs and glycoligand sequence in RosettaCarbohydrate format. The corresponding 62 unbound protein receptor crystal structures used to create the unbound benchmark target set are also reported, including the $\mathrm{C} \alpha$-RMSD of the unbound protein backbone to the bound.

\section{Metadata}

- PDB_ID

The four-letter PDB code for the bound protein-glycoligand crystal structure

- Protein_Type

$\bigcirc$ The identity of the protein receptor. Options include Antibody, Carbohydrate-binding module (CBM), Enzyme, Lectin/glycan-binding protein (GBP), Viral (e.g., hemagglutinin)

- Resolution

○ The resolution of the bound crystal structure in $\AA$

- Protein_Chains

○ The chain IDs of the crystal structure kept for the protein receptor (i.e., all coordinates for all other protein chains in the crystal structure, if present, are discarded)

- Glycoligand_Chain

○ The chain ID of the crystal structure kept for the glycoligand (i.e., all coordinates for all other glycoligand chains in the crystal structure, if present, are discarded)

- Glycoligand_Sequence

$\circ$ The full primary sequence of the glycoligand written in RosettaCarbohydrate format

- Unbound_PDB

$\bigcirc \quad$ The four-letter PDB code for the unbound protein crystal structure

- Ca_RMSD

- Note: "-" denotes that no unbound protein crystal structure was available

○ The $\mathrm{C} \alpha$-RMSD of the bound to unbound protein-receptor backbone in $\AA$ (calculated using PyMOL)

- FTMap_CHX_Probe_Success

- Note "-" denotes that no unbound protein crystal structure was available

○ Did the aligned unbound protein structure result in a CHX probe from FTMap in the known glycoligand binding site (as determined by visual inspection)?

- True: FTMap predicted a CHX probe within the known binding site

- False: FTMap did not predict a CHX probe within the known binding site 
Table_S3.xls: Detailed GlycanDock $\left\langle N_{5}\right\rangle$ docking performance on the set of 109 bound benchmark targets using input starting structures of increasing initial ring-RMSD.

\section{Metadata}

- PDB_ID

The four-letter PDB code for the bound protein-glycoligand crystal structure

- initial_ring_RMSD

○ The initial ring-RMSD bin of the input starting structures to GlycanDock (e.g., 3 means the $3.0 \pm 0.1 \AA$ initial ringRMSD bin)

- Ranges from 0 (crystal structure as input) to 10

- N_near_native

○ The total number of near-native (<2 Å ring-RMSD) models sampled by GlycanDock, regardless of score

- Can range from 0 to 2000

- N5

- The number of near-native models ranked within the 5-top-scoring by interface score (reported as $N_{5}$ in the manuscript)

- Can range from 0 (worst) to 5 (best)

- 0 means no near-native models were ranked within the 5-top-scoring

- 5 means all five of the 5-top-scoring models were near-native

- bootstrap_N5_mean

○ The bootstrap ensemble average of $N_{5}$ per target per initial ring-RMSD bin (reported as $\left\langle N_{5}\right\rangle$ in the manuscript)

- Can range from 0.0 (worst) to 5.0 (best)

- bootstrap_N5_std

$\circ$ The standard deviation of the bootstrap ensemble average of $N_{5}$ described above (mentioned as $\sigma\left\langle N_{5}\right\rangle$ in the manuscript) 
Table_S4.xls: Detailed GlycanDock $\left\langle N_{5}\right\rangle$ docking performance on the set of 62 unbound benchmark targets using input starting structures of increasing initial ring-RMSD.

\section{Metadata}

- PDB_ID

- The four-letter PDB code for the unbound protein crystal structure

- initial_ring_RMSD

○ The initial ring-RMSD bin of the input starting structures to GlycanDock (e.g., 3 means the $3.0 \pm 0.1 \AA$ initial ringRMSD bin)

- Ranges from 1 to 10

- N_near_native

○ The total number of near-native (<2 Å ring-RMSD) models sampled by GlycanDock, regardless of score

- Can range from 0 to 2000

- N5

- The number of near-native models ranked within the 5-top-scoring by interface score (reported as $N_{5}$ in the manuscript)

- Can range from 0 (worst) to 5 (best)

- 0 means no near-native models were ranked within the 5-top-scoring

- 5 means all five of the 5-top-scoring models were near-native

- bootstrap_N5_mean

○ The bootstrap ensemble average of $N_{5}$ per target per initial ring-RMSD bin (reported as $\left\langle N_{5}\right\rangle$ in the manuscript)

- Can range from 0.0 (worst) to 5.0 (best)

- bootstrap_N5_std

$\circ$ The standard deviation of the bootstrap ensemble average of $N_{5}$ described above (mentioned as $\sigma\left\langle N_{5}\right\rangle$ in the manuscript) 
Table_S5.xls: Detailed results and biophysical features of all the 50-top-scoring models per target per initial ring-RMSD bin (not including interfacial hydrogen bonds).

\section{Metadata}

- PDB_ID

- The four-letter PDB code for the bound protein crystal structure

- initial_ring_RMSD

- The initial ring-RMSD bin of the input starting structures to GlycanDock (e.g., 3 means the $3.0 \pm 0.1 \AA$ initial ringRMSD bin)

- Ranges from 0 (crystal structure as input) to 10 ring_RMSD

$\circ$ The ring-RMSD in $\AA$ of the model

- The closer to 0 the better

- $\quad$ ring_SRMSD

○ The ring-SRMSD in $\AA$ of the model

- The closer to 0 the better

- interface_score

- The Interface Score in REU of the model

- More negative is better

- dG_separated

$\circ$ The dG separated score in REU of the model, as calculated by the InterfaceAnalyzerMover

- This is similar to Interface Score, but when the protein-glycoligand is separated, packing of all side-chain rotamers occurs

- More negative is better

- n_intf_res_res_contacts

$\circ$ The number of interfacial residue-residue contacts in the model

- n_nat_intf_res_res_contacts

$\circ$ The number of interfacial residue-residue contacts in the native crystal structure recovered in the model

- n_intf_res_res_contacts may be larger if non-native interfacial residue-residue contacts are made in the model

- Fnat_res_res_contacts

- The recovery of native interfacial residue-residue contacts in the model

- The fraction ranges from 0.0 (no native contacts recovered) to 1.0 (all native contacts recovered) 
- n_intf_residues

- The number of residues present at the interface in the model

- n_nat_intf_residues

- The number of residues present at the interface in the model that are also present in the native crystal structure

- n_intf_residues may be larger if non-native interface residues are present in the model

- Fnat_intf_residues

○ The recovery of native interface residues in the model

- The fraction ranges from 0.0 (no native residues recovered) to 1.0 (all native residues recovered)

- dSASA_intf

○ The solvent accessible surface area (SASA) buried at the interface in $\AA^{2}$, as calculated by the InterfaceAnalyzerMover

- dSASA_hphobic

○ The hydrophobic portion of dSASA_intf, as calculated by the InterfaceAnalyzerMover

- dSASA_polar

O The polar portion of dSASA_intf, as calculated by the InterfaceAnalyzerMover

- packstat

- Rosetta's packing statistic score for the interface, as calculated by the InterfaceAnalyzerMover

- Ranges from 0.0 (bad packing at the interface) to 1.0 (perfect packing at the interface) 
Table_S6.xls: Detailed information on interfacial hydrogen bonds of all the 50-top-scoring models per target per initial ringRMSD.

\section{Metadata}

- PDB_ID

- The four-letter PDB code for the bound protein crystal structure

- initial_ring_RMSD

○ The initial ring-RMSD bin of the input starting structures to GlycanDock (e.g., 3 means the $3.0 \pm 0.1 \AA$ initial ringRMSD bin)

- glycolig_residue

- The specific residue of the glycoligand unit making (or not making) interfacial hydrogen bonds

- Reported as <PDB residue number $><$ chain ID $>$ (e.g., 118X, glycoligand residue 118 chain X)

- interface_hbonds

$\bigcirc$ The interfacial hydrogen bond(s) being made by this glycoligand residue

- Format: <protein residue number $1><$ protein chain $1>_{-}<$protein residue number $2><$ protein chain $2>$

- For example, 73A_148A means that this particular glycoligand residue is making an interfacial hydrogen bond with protein residue 73 and 148 of chain $A$

- If the glycoligand residue is not making any interfacial hydrogen bonds, it is reported as NaN 
Primary Rosetta Movers used for glycoligand sampling and optimization during Stage 2 of GlycanDock

The GlycanDock algorithm employs multiple Rosetta Movers that are randomly selected at different probabilities to perform glycoligand conformational sampling. Default GlycanDock behavior consists of performing eight inner cycles of rigid-body sampling and optimization and eight inner cycles of glycosidic torsion sampling and optimization (performed in either order for 10 total outer cycles; see Figure 1).

For glycoligand rigid-body refinement, the RigidBodyPerturbMover (rotation $=7.5^{\circ}$, translation $=0.5 \AA$ ) is employed with probability 0.67 ; the same RigidBodyPerturbMover followed by FaDockingSlideIntoContact is employed with probability 0.33 .

For glycosidic torsion angle refinement, the SugarBBSampler is employed with probability 0.45 ; the SmallBBSampler is employed with probability 0.3 (sampling at uniform distributions of $\pm 15^{\circ}, \pm 45^{\circ}$, and $\pm 90^{\circ}$ at a probability ratio of $4: 2: 1$, respectively); the ShearMover is employed with probability 0.2 ; and the RingPlaneFlipMover is employed with probability 0.05 . If GlycanDock is called with the -refine_only flag, only the $\pm 15^{\circ}$ bin of the SugarBBSampler is employed (still with total probability 0.3).

Interfacial rotamer optimization is employed after each rigid-body and glycosidic torsion angle sampling move, whereas full-structure energy minimization is employed after every other sampling move. Rotamer optimization consists employing the EnergyCutRotamerTrialsMover (energycut $=0.05$ ) after seven of the eight sampling moves followed by the PackRotamersMover (using the RestrictToInterface task and an interface distance of $16 \AA$ ) after the eighth sampling move. Minimization consists of employing the MinMover (minimization type $=$ "lbfgs_armijo_nonmonotone", tolerance $=$ $0.01)$.

\section{ShearMover}

A shearing motion is a movement that minimizes the downstream effect of a torsional change by making a counter-rotation that results in limiting downstream moves to small translations. For a geometrical shearing motion of downstream coordinates to occur, the two twisting bonds must be near parallel, and the bond twists must occur in opposite directions with equal magnitude. If the bonds are not near parallel, the downstream chain will spiral. In the case of peptides, because the $\omega$ angle is nearly always a trans peptide bond, the two bonds on either side, that is, $\psi_{n-1}$ and $\varphi_{n}$, are forced to be near parallel.

Traditionally, shear moves in Rosetta have simply made an equal but opposite twist to the $\psi_{n-1}$ and $\varphi_{n}$ of a peptide pose. In non-peptide cases (e.g., polysaccharides), in the absence of a trans peptide bond, there is no guarantee of any particular bonds being near-parallel to each other, so functions were written to search for nearby bonds with similar directional orientations. This modification to the underlying code has permitted shearing moves to be made during sampling strategies of polysaccharides in Rosetta. Furthermore, special checks needed to be added to ensure that a pair of shearing torsional changes were not made across 
a branch point. Otherwise, a saccharide main chain might shear while its branch or branches twisted.

\section{RingPlaneFlipMover}

Typically, a shearing movement involves a small angle value change. However, in the special but common case of chains of equatorial $(1 \beta \rightarrow 4)$ glycosidic linkages for aldopyranoses or $(2 \beta \rightarrow 5)$ linkages for ketopyranoses, a full $180^{\circ}$ rotation can be made at the anomeric bond with a counter move at the linkage position. Such a shearing motion effectively "flips over" a single carbohydrate ring relative to the orientation of the other rings in the carbohydrate chain. It is expected that allowing GlycanDock to sample conformations with such flipped rings will help in cases where an oligo- or polysaccharide is "stuck" with its ring plane in the wrong orientation, with substituents trapped on the wrong side of the ring. Thus, we have added such a sampling method to GlycanDock that is limited to systems with relevant aldoor ketopyranoses. 
Selection and preparation of benchmark set of protein-glycoligand complexes

When multiple glycoligands were present in a bound structure, only the most well-resolved glycoligand as measured by average B-factor was kept. In cases where duplicate chains were resolved in a bound structure, only the protein chains within $6 \AA$ of any atom of the most well-defined glycoligand were kept. For unbound structures with duplicate chains, only the protein chains relevant for glycoligand binding that aligned best with the respective bound structures were kept. Water and solvent molecules were removed, missing residues were ignored, coordinates for the first residue conformation were kept in cases where alternative conformations were reported, and all three-letter PDB codes for the carbohydrate residues were replaced with the equivalent three-letter code from the Rosetta/IUPAC naming system $^{2}$. Ions (e.g., calcium, sodium, magnesium) were kept, except those resolved in the glycoligand binding site of unbound structures, in which case the ion was removed. For consistency, the glycoligand was given a unique chain identifier (chain " $\mathrm{X}$ ") different from the chain identifiers of its protein receptor (e.g., chains "A" and "B"). Structures in which the protein was identified as chain " $\mathrm{X}$ " were given a different chain identifier so that only the glycoligand was chain " $X$ ". Finally, the text lines containing the atomic coordinates the glycoligand were moved to the bottom of the PDB file to ensure proper arrangement of the Rosetta FoldTree-a directed, acyclic, connected graph which handles the kinematic propagation of sampling perturbations ${ }^{3}$. The resulting structure for each bound and unbound target was used as the reference native structure for measuring the quality of GlycanDock models. Processed PDB files and the script to perform the described processing procedure can be provided upon request. See Table S2 for additional details on the protein structures and glycoligands employed in this work. 
Python pseudo-code for calculating the bootstrap ensemble average $\left\langle N_{5}\right\rangle$

import pandas as pd

import numpy as np

metric = "ring_RMSD"

cutoff $=2.0$ \# 2 Angstroms, near-native model cutoff

score = "interface score" \# in units of REU

topX $=5$ \# consider the 5-top-scoring

n_samples $=1000$ \# the number of models to randomly sample from the set

$\mathrm{n}$ repeats $=5000$ \# the number of times to randomly resample form

the set

def calc Nsuccess (df):

return sum(df.sort_values (score).iloc[:topX][metric] < cutoff)

def random resample (df):

return df.sample(replace=True, $n=n$ samples)[[metric,

score] ].reset_index (drop=True)

bootstrap ensemble $=$ np.arrary ([])

for ii in xrange (n_repeats):

bootstrap_ensemble $=$ np. append (bootstrap_ensemble,

calc_Nsuccess(random_resample(df)))

Where $\mathrm{df}$ is a pandas DataFrame that, at minimum, contains all the ring-RMSDs and Interface Scores for each model generated. 


\section{"Blind"-like docking pipeline using FTMap and RosettaLigand}

\section{Using FTMap to predict glycoligand binding sites}

FTMap was accessed using its web-based server via http://ftmap.bu.edu/login.php. Each of the 62 unbound protein structures from the unbound benchmark set were used as input to FTMap. Specifically, the unbound protein structures used as input were those that were cleaned and aligned to the bound structure as described in the benchmark set preparation section in Materials and Methods. FTMap requires only an input protein structure (or a PDB code) and the relevant chain(s) to be analyzed. Default FTMap settings were used (i.e., the Advanced Settings were untouched). FTMap uses various small organic molecules probes (e.g., acetamide, benzene, isobutanol, cyclohexane, ethanol, urea) to extensively sample the surface of the input protein in search for potential ligand binding "hot spots".

In this work, we specifically looked at the output locations of the cyclohexane (CHX) probes due to its structural similarity to a carbohydrate. Given that the glycoligand binding site was known, we used visual inspection to determine if FTMap correctly identified the glycoligand binding site with at least one CHX probe. If multiple CHX probes were predicted in or near the known glycoligand binding site, the probe with a center-of-mass closest to the center-ofmass of the bound glycoligand was used for RosettaLigand docking. All center-of-mass calculations were performed using PyMOL's centerofmass command. FTMap predicted the known glycoligand binding site using a CHX probe for 36 of the 62 unbound protein structures. Therefore, 36 unbound targets were docked using RosettaLigand. The 36 PDB targets are listed in Table S1.

\section{Generation of glycoligand conformers for RosettaLigand docking}

The Rosetta GlycanSampler algorithm (with default settings) was used to generate 200 conformations of each of the 36 glycoligands. However, at the time of writing, the GlycanSampler algorithm is unpublished. Briefly, GlycanSampler uses repeated cycles of glycan conformational sampling, side-chain rotamer packing, and energy minimization to produce low-scoring glycan structures. Many of the components of GlycanSampler glycan conformational sampling were incorporated into the glycoligand conformational sampling of the GlycanDock algorithm.

To truly emulate "blind" docking, we built a single de novo structure each of the 36 glycoligands to serve as the input to GlycanSampler. De novo glycoligand structures were generated using the pose_from_saccharide_sequence Rosetta application, where the glycoligand sequence was provided via the -carbohydrates: saccharide_sequence flag. The coordinates for the de novo glycoligand conformation is written to a file called out_saccharide.pdb, and the glycoligand is assigned as chain " $X$ ". The pose_from_saccharide_sequence application performs one round of energy minimization where glycosidic torsion angles and side-chain rotamers of the glycoligand are treated as a degree-of-freedom. Notably, energy minimization includes the sugar_bb scoring term, which helps ensure that the glycosidic torsion angles are energetically realistic for a carbohydrate chain. So, while no glycosidic torsion angle sampling occurs within the pose_from_saccharide_sequence application, this approach can be used as an alternative to GlycanSampler for generating de novo glycoligand conformations. 
Example command to generate a single de novo conformation of the glycoligand from bound target PDB 154L:

./pose_from_saccharide_sequence.macosclangrelease -include_sugars - carbohydrates: saccharide_sequence "b-D-GlcpNAc-(1->4)-b-D-GlcpNAc-(1$>4)$-b-D-GlcpNAc” -nstruct 1

Another suggested approach would be using BCL::Conf ${ }^{4}$, a freely accessible small molecule conformational sampling package available as a part of the BCL. The BCL (Biology and Chemistry Library project; http://meilerlab.org/bclcommons) contains many useful tools for performing and analyzing molecular simulations (many of which are complementary to the Rosetta software suite).

To compare to GlycanSampler performance, we also used the BCL to generate 200 initial conformations for each of the 36 unbound target glycoligands. BCL glycoligand models had an average heavy-SRMSD of $2.03 \AA \pm 0.98 \AA$ (minimum $0.17 \AA$, maximum $7.62 \AA$ ), where heavy-SRMSD is calculated using all glycoligand heavy-atoms after superposition of the model glycoligand onto the native bound glycoligand. Accordingly, GlycanSampler produced glycoligand models that were on average about 0.5 Å heavy-SRMSD more accurate than BCL models.

\section{Converting glycoligand structures to RosettaLigand-compatible inputs}

We first converted the native crystal structures to a RosettaLigand compatible format. First, the "ATOM" lines defining each carbohydrate residue of the glycoligand needed to be converted into an .sdf formatted file. This was accomplished by copying the "ATOM" lines of the glycoligand from the native crystal structure into a separate file (e.g., 154L-glycolig.pdb) and converting it to an .sdf file using Open Babel5:

\section{./obabel 154L-glycolig.pdb -0 154L-glycolig.sdf}

We then converted each .sdf file into a RosettaLigand-compatible .pdb and accompanying .params file using Rosetta molfile_to_params.py tool:

Rosetta/main/source/scripts/python/public/./molfile_to_params.py 154Lglycolig.sdf -n LG1 -p 154L-glycolig-RL.pdb

This converts the.sdf file into a new .pdb file that now defines the glycoligand as a single, non-oligomeric molecule with residue name LG1. The accompanying params files defines each atom type, bond type, internal geometries, and side chain torsions of the ligand. Using the command-line, ligand .pdb file is then combined with the "ATOM" lines from the native crystal structure defining the protein receptor to create the RosettaLigand-compatible native protein-(glyco)ligand complex.

cat 154L-protein.pdb 154L-glycolig-RL.pdb > 154L-native-RL.pdb 
The same approach was taken to create the RosettaLigand compatible formats for the de novo glycoligand structures. However, since we generated 200 conformations per glycoligand (rather than the single conformation needed for the native crystal structure), the params file was made aware of multiple glycoligand conformers.

\section{Running RosettaLigand for "blind"-like protein-glycoligand docking}

We ran RosettaLigand using the default settings as used by the ROSIE server 6 . An example $\mathrm{XML}$ script to run RosettaLigand is shown below (note there are four variables, $\langle\mathrm{W}\rangle,\langle\mathrm{X}\rangle$, $\langle\mathrm{Y}\rangle$, and $\langle\mathrm{Z}\rangle$ ). $\langle\mathrm{W}\rangle$ is the width of the scoring grid, which we set to $6 \times$ the length of the glycoligand (e.g., a tetrasaccharide would give $\langle\mathrm{W}\rangle=24$ ). $\langle\mathrm{X}\rangle,\langle\mathrm{Y}\rangle$, and $\langle\mathrm{Z}\rangle$ are the $\mathrm{x}, \mathrm{y}$, and $\mathrm{z}$ components, respectively, of the starting location of the glycoligand (i.e., the XYZ coordinate to which the center-of-mass of the glycoligand is translated). The $\langle\mathrm{X}\rangle,\langle\mathrm{Y}\rangle$, and $\langle\mathrm{Z}\rangle$ values are set using the center-of-mass of the chosen cyclohexane probe predicted by FTMap.

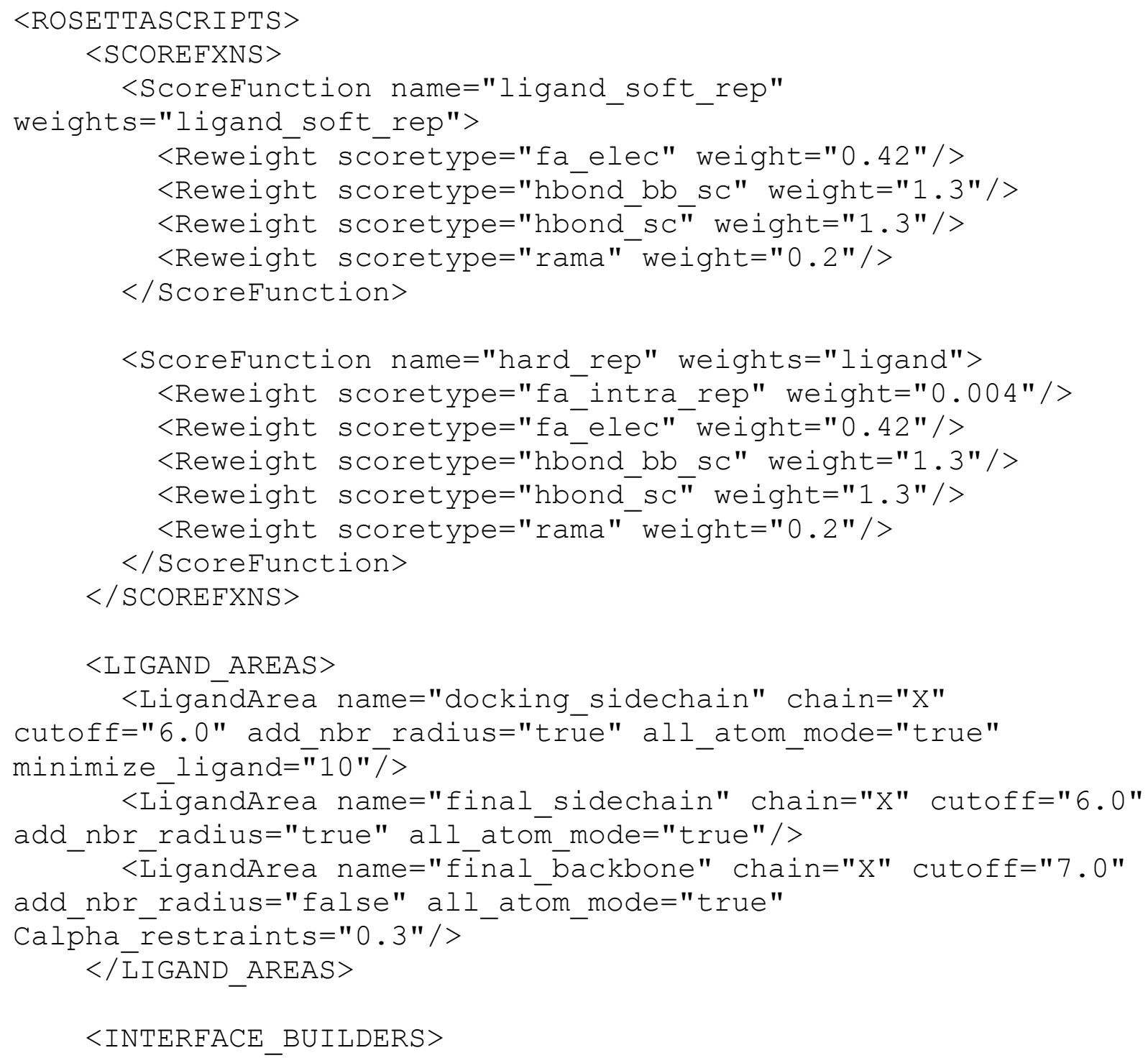




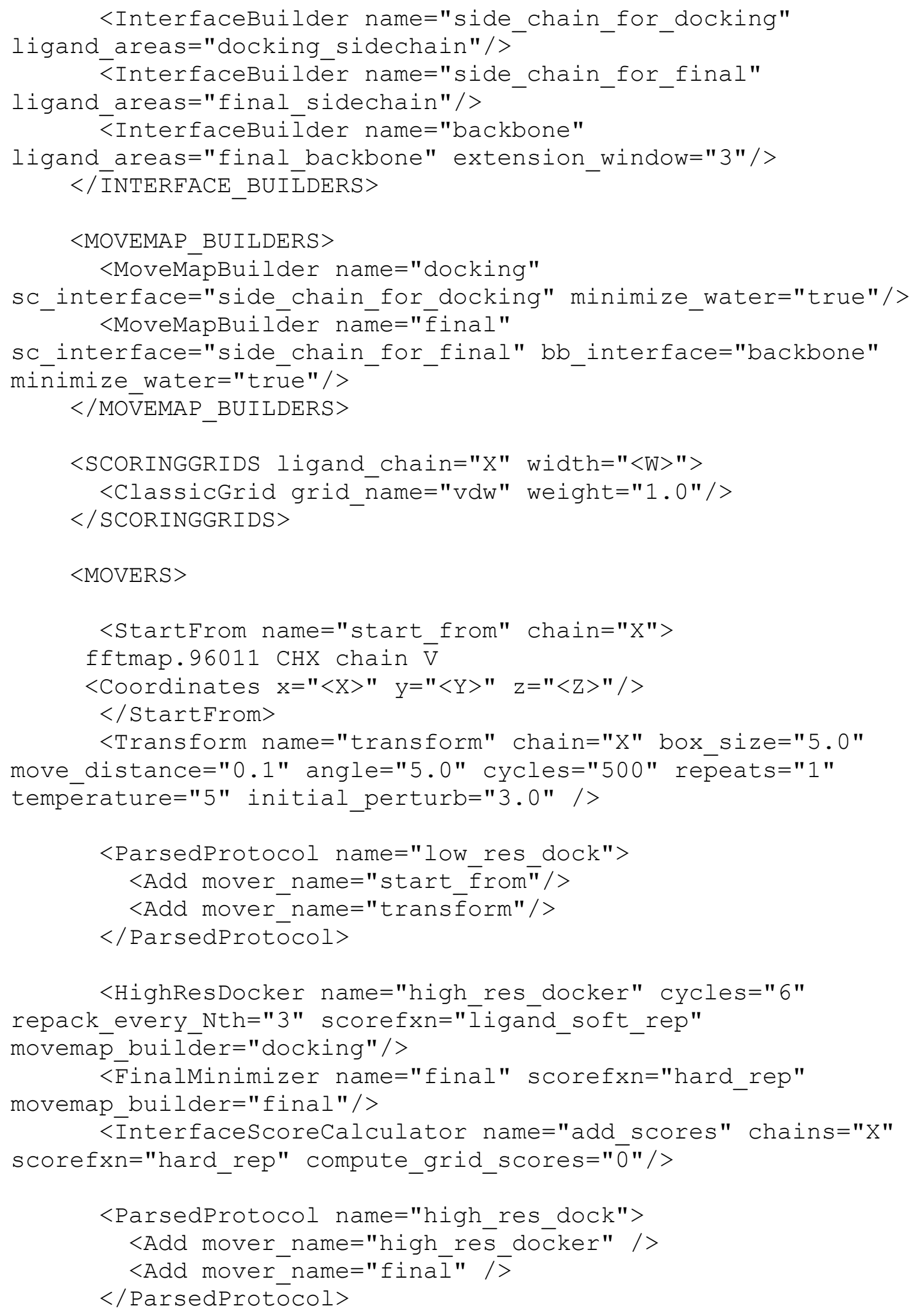




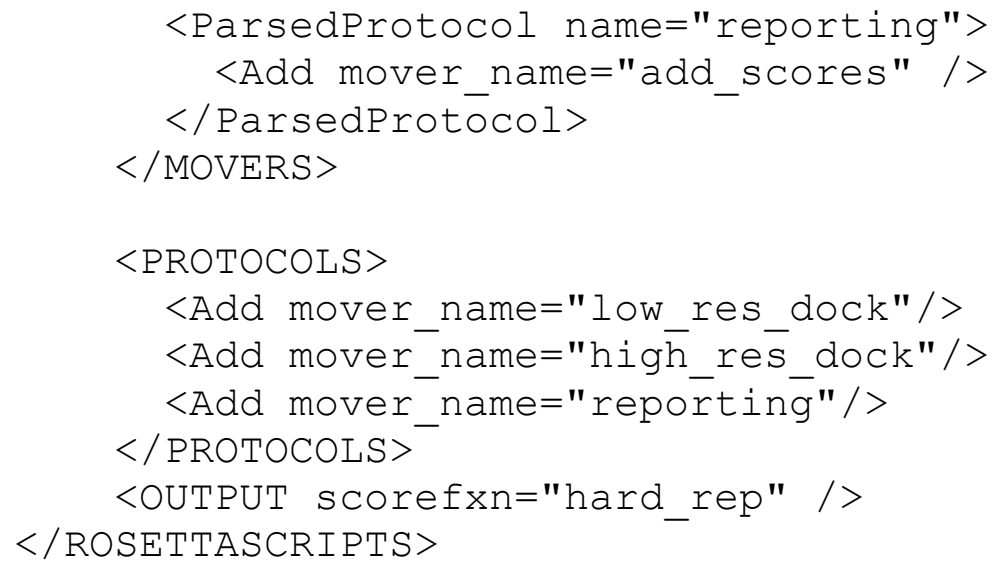

\section{References}

(1) Waskom, M.; the seaborn development team. Mwaskom/Seaborn. Zenodo September 2020. https://doi.org/10.5281/zenodo.592845.

(2) Labonte, J. W.; Adolf-Bryfogle, J.; Schief, W. R.; Gray, J. J. Residue-Centric Modeling and Design of Saccharide and Glycoconjugate Structures. J. Comput. Chem. 2017, 38 (5), 276-287. https://doi.org/10.1002/jcc.24679.

(3) Wang, C.; Bradley, P.; Baker, D. Protein-Protein Docking with Backbone Flexibility. J. Mol. Biol. 2007. https://doi.org/10.1016/j.jmb.2007.07.050.

(4) Kothiwale, S.; Mendenhall, J. L.; Meiler, J. BCL::Conf: Small Molecule Conformational Sampling Using a Knowledge Based Rotamer Library. J. Cheminform. 2015, 7 (1), 47. https://doi.org/10.1186/s13321-015-0095-1.

(5) O’Boyle, N. M.; Banck, M.; James, C. A.; Morley, C.; Vandermeersch, T.; Hutchison, G. R. Open Babel: An Open Chemical Toolbox. J. Cheminform. 2011, 3 (10), 1-14. https://doi.org/10.1186/1758-2946-3-33.

(6) Lyskov, S.; Chou, F. C.; Conchúir, S. Ó.; Der, B. S.; Drew, K.; Kuroda, D.; Xu, J.; Weitzner, B. D.; Renfrew, P. D.; Sripakdeevong, P.; Borgo, B.; Havranek, J. J.; Kuhlman, B.; Kortemme, T.; Bonneau, R.; Gray, J. J.; Das, R. Serverification of Molecular Modeling Applications: The Rosetta Online Server That Includes Everyone (ROSIE). PLoS One 2013, 8 (5), 63906. https://doi.org/10.1371/journal.pone.0063906. 